\title{
Antimicrobial Resistance and The Surgical Renaissance
}

\author{
R Harricharan \\ Medical Student, University of KwaZulu-Natal \\ Corresponding author: Rivona Harricharan (rivona.h3@gmail.com)
}

Envision a future where patients are cared for without the dread of hospital-acquired infections and antimicrobial resistance. Is this notion a highly fantasised illusion of clinicians or is it possible to attain such an ambition?

The ultimate goal of surgery is to use operative techniques to repair, remove or replace any damaged or diseased part of the body to provide a better prognosis for the patient. To fully attain this objective, antibiotics have been instrumental in the prevention and treatment of infections. Antibiotics function through exerting their action on the intracellular signalling pathways of bacteria, subsequently inhibiting their growth. Consequently, antibiotics have inadvertently facilitated the worldwide increase in antimicrobial resistance. Antimicrobial resistance encompasses the evolution of bacterial defence mechanisms enabling them to escape the effect of antibiotics. Patient adherence and inappropriate use of antibiotics have contributed towards antibiotic resistance. There is undisputable evidence to support the association between antimicrobial resistance leading to higher morbidity and mortality rates. Due to various factors (natural and financial), the quantity of novel antibiotics in the past decade has been diminishing. The process of new antibiotic development is an expensive and long one, further exacerbating the need for suitable alternatives to antimicrobial agents.

Antibiotics are vital to surgery and postoperative care. Therefore, the consequence of antimicrobial resistance has a profoundly adverse impact on both surgery and postoperative care globally. Previous studies have emphasised the poor surgical outcomes related to antimicrobial resistance. ${ }^{1}$ Simple surgical procedures could become more risky and dangerous without the use of effective antimicrobial agents. Antimicrobial resistant patients requiring transplant surgeries or if they are immunocompromised are the most vulnerable and susceptible to detrimental outcomes. ${ }^{2}$ The fate of surgeries in antimicrobial-resistant individuals appears doomed unless suitable interventions are established and implemented.

Since antimicrobial resistance poses a global public health threat, the Global Antibiotic Research and Development Partnership was established by the World Health Organization and the Drugs for Neglected Diseases initiative. The initiative focuses on four key areas. The first focuses on antibiotic resistance in the treatment of sexually-transmitted infections. The second, the antimicrobial memory recovery and exploratory programme aims to identify new drug possibilities obtained from information of previously abandoned antibiotic development projects. The third area of focus involves the formation of new treatment regimens for neonates with sepsis. Finally, the paediatric antibiotic platform aims to enhance current treatments and accelerate the development of new antibiotics. Unfortunately, antibiotic resistance related to surgery is not a key area of focus.

Therefore, a multi-modal approach is warranted at various levels for surgery to be effective for patients exhibiting antimicrobial resistance:

- prophylactic approach

- $\quad$ prevention and surveillance measures

- $\quad$ education, guidelines and policies

- postoperative use of antibiotics and use of automated systems

- genetic therapy

Conventional surgical interventions include improving hygiene (related to operating theatres, equipment, surgeons and nurses) with focus on appropriate hand-washing regimes. A study by Huh et al. evaluated the practice of monitoring the use of surgical prophylactic antibiotics. ${ }^{3}$ The parameters assessed were the duration of administration for the antibiotic, the time at which they were administered, in addition to the relevance of the course of antibiotic therapy, and also random screening for adherence rates. The study also evaluated the implementation of computerized digital support systems (Samsung Antibiotic Prescription System). ${ }^{3}$ The system requires the physician to enter the patient's medical record and select the purpose for the use of the antibiotic. The system displayed the potential list of microorganisms that may be responsible and assisted in providing antibiotic recommendations. Too often, antibiotics are overprescribed for hospital acquired infections (surgical site infections, pneumonia, urinary tract infections). With the assistance of such automated systems, surgeons will be able to prescribe with higher accuracy, whilst simultaneously protecting antibiotic resources. This may augment efficacious use of antimicrobial therapy at both operative and postoperative stages of patient care.

Large scale implementation of computerised digital support systems may be highly improbable. Their principle function 
can be condensed into the development of national guidelines for each country depending on their resources available and the most common resistant infections affecting that country. This set of guidelines needs to be updated at least on an annual basis. All surgical-related disciplines should include a focus on antimicrobial resistance, guidelines and prescribing in their residency training programs. Some ideas that have been suggested in previous studies include the establishment of hospital-acquired infection preventative and promotion strategies, in addition to novel barriers to prevent patient-topatient transmission and antimicrobial coated devices that patients would usually share. ${ }^{1}$ More advanced surveillance tools are required to improve the rate at which microbiological outbreaks are reported and the response at which hospitals attempt to control the infection.

Other quicker more promising approaches may include manipulation, editing or silencing of bacterial genes. The use of peptide-conjugated phosphorodiamate morpholino oligomers could in future become a standardised practice in humans. Peptide-conjugated phosphorodiamate morpholino oligimers prevent the translation of resistance genes thereby enhancing antibiotic efficacy. ${ }^{4-5}$ Such ideas, even if plausible, have their disadvantages when attempting to translate them into clinical settings. Another significant challenge is how we can address this problem in lower to middle income countries which have the greatest burden yet minimal reserves and basic amenities.

I anticipate that over the next decade we shall expand our minds in attempting to explore the possible solutions to antimicrobial resistance. It is the endeavour of both clinicians and scientists to save the lives of as many patients as they can through surgical interventions and pharmacological developments. Antimicrobial resistance is an example of Darwin's theory of natural selection, highlighting the subtle yet complex interplay between humans and bacteria. One day in the distant future it may drive the evolutionary mechanisms enabling the human population to develop more resilient genes similar to patients unaffected by antimicrobial resistance. Those unaffected by antimicrobial resistance live longer and pass on these traits to their offspring and slowly we drift into a slightly more evolved species. Either way, the future of surgery is dependent on how we view, respond and adapt to antimicrobial resistance.

\section{REFERENCES}

1. Harris A, Pineles L, Perencevich E. Recognising the value of infection prevention and its role in addressing the antimicrobial resistance crisis. BMJ Qual Saf. 1 Aug 2017;26(8):683-6.

2. Friedman ND, Temkin E, Carmeli Y. The negative impact of antibiotic resistance. Clinical Microbiology and Infection. 31 May 2016;22(5):416-22.

3. Huh K, Chung DR, Park HJ, Kim MJ, Lee NY, Ha YE, et al. Impact of monitoring surgical prophylactic antibiotics and a computerized decision support system on antimicrobial use and antimicrobial resistance. American journal of infection control. 1 Sep 2016;44(9):e145-52.

4. Ayhan DH, Tamer YT, Akbar M, Bailey SM, Wong M, Daly $\mathrm{SM}$, et al. Sequence-specific targeting of bacterial resistance genes increases antibiotic efficacy. PLoS Biology. 15 Sep 2016;14(9):e1002552.

5. Howard JJ, Sturge CR, Moustafa DA, Daly SM, Marshall-Batty $\mathrm{KR}$, Felder CF, et al. Inhibition of Pseudomonas aeruginosa by peptide-conjugated phosphorodiamidate morpholino oligomers. Antimicrobial Agents and Chemotherapy. 1 Apr 2017;61(4):e01938-16.

\section{Author's biography}

Rivona Harricharan is a second-year medical student at the University of KwaZulu-Natal. In 2017, she became a member of the Golden Key International Honour Society. Miss Harricharan graduated with a Masters degree in Medical Sciences Summa cum laude and aspires to complete her $\mathrm{PhD}$. Her research focuses on understanding the complex relationship between addiction and inflammation. She has recently joined the Centre for AIDS research in South Africa as part of their placement programme. She demonstrates excellently her ability to work on multiple projects simultaneously as she is also involved in traumatic brain injury research at the Department of Neurosurgery in Inkosi Albert Luthuli Chief Hospital. In addition, Miss Harricharan is passionate about cancer awareness and community education which is exemplified through her role in the Woodview Cancer Support Group. When she is not busy with medical school and her research activities, Miss Harricharan enjoys extreme outdoor activities and painting.

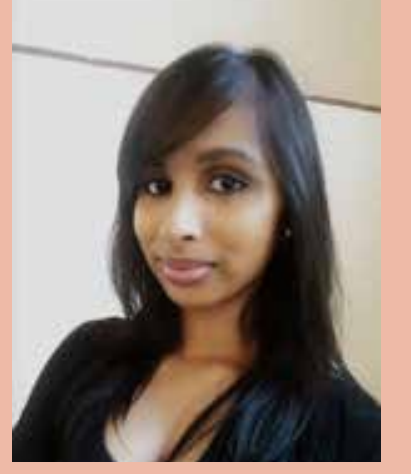

